\title{
Uso de la bicicleta como transporte alternativo: implicaciones de la cuestión cultural en la preservación del medio ambiente
}

\section{Uso da bicicleta como transporte alternativo: implicações da questão cultural na preservação do meio ambiente}

Use of bicycles as alternative transport: implications of the cultural question in the preservation of the environment

Adalci dos Anjos Ferreira ${ }^{1}$ Danila Fernanda Rodrigues Frias ${ }^{2}$

${ }^{1}$ Mestre em Ciências Ambientais pela Universidade Brasil, Fernandópolis, São Paulo. Possui graduação em Ciências pela Faculdade de Educação Ciências e Letras de Uberlândia e Especialização em Supervisão Escolar pela Faculdade Católica de Uberlândia. E-mail: adalci@ufu.br, Orcid: https://orcid.org/0000-0001-6890-1874.

${ }^{2}$ Doutor e mestre em Medicina Veterinária pela Universidade Estadual Paulista Júlio de Mesquita Filho (UNESP). Graduado em Medicina Veterinária pela Universidade Brasil. Professor titular do Curso de Mestrado em Ciências Ambientais, Universidade Brasil, Fernandópolis, São Paulo.

E-mail: danila.frias@universidadebrasil.edu.br, Orcid: https://orcid.org/ 0000-0001-8621-3338. 
Resumen: Fue analizada la cultura de la utilización de la bicicleta como medio de transporte en el municipio de Uberlândia. Se realizó el levantamiento de datos por medio de la observación de documentos e informaciones del Secretaría de Tráfico y Transportes, Universidad Federal de Uberlândia y Ayuntamiento local. También se aplicó un cuestionario electrónico, con participación de 109 ciclistas. La falta de infraestructura es un grave problema en el municipio, que posee cerca de 98.119 metros de ciclovías y tiene alto índice de accidentes que involucran a los ciclistas, lo que hace que la adhesión al uso de la bicicleta sea disminuida. Se concluye que la deficiencia de infraestructura cicloviaria ha ocasionado número elevado de accidentes. De esta forma, se acentuó la necesidad de discusiones sobre acciones que apunten a aumentar el uso de la bicicleta y la necesidad de implementar medidas para disminuir las ocurrencias de accidentes de tránsito involucrando a ciclistas.

Palabras clave: accidentes de tránsito; ciclistas; movilidad urbana; sostenibilidad.

Resumo: Analisou-se a cultura da utilização da bicicleta como meio de transporte no município de Uberlândia. Realizou-se levantamento de dados por meio da observação de documentos e informações da Secretaria de Trânsito e Transportes, Universidade Federal de Uberlândia e Prefeitura local. Também foi aplicado um questionário eletrônico, com participação de 109 ciclistas. A falta de infraestrutura é um grave problema no município, que possui cerca de 98.119 metros de ciclovias e tem um alto índice de acidentes envolvendo ciclistas, o que faz com que a adesão ao uso da bicicleta seja diminuída. Conclui-se que a deficiência de infraestrutura cicloviária ocasionou um número elevado de acidentes. Desta forma, ficou acentuada a necessidade de discussões sobre ações que visem aumentar o uso da bicicleta e a necessidade de implantar medidas para diminuir as ocorrências de acidentes de trânsito envolvendo ciclistas.

Palavras-chave: acidentes de trânsito; ciclistas; mobilidade urbana; sustentabilidade.

Abstract: It was analyzed the culture of bicycle use as a means of transportation in the municipality of Uberlândia. Data was gathered through the observation of documents and information from Secretariat of Transit and Transport, Federal University of Uberlândia, and the local City Hall. An electronic questionnaire was also applied, with the participation of 109 cyclists. The lack of infrastructure is a serious problem in the municipality, which has about 98,119 meters of bikeways with a high rate of accidents involving cyclists, which means that the use of the bicycle is reduced. It is concluded that the lack of cycling infrastructure has caused a high number of accidents. In this way, the need for discussions on actions aimed at increasing bicycle use and the need to implement measures to reduce the occurrence of traffic accidents involving cyclists were highlighted.

Keywords: traffic accidents; cyclists; urban mobility; sustainability. 


\section{INTRODUCCIÓN}

Actualmente la utilización de vehículos automotores es la preferencia de la mayoría de las personas en los grandes centros urbanos, pues trae flexibilidad de horario, libertad de trayecto y confort en la locomoción cotidiana, tales comodidades no se aplican al transporte colectivo.

Con el crecimiento de las ciudades y la falta de planificación urbana, la infraestructura vial no soporta eficientemente la circulación de coches y autobuses en gran escala, y no es posible una reestructuración urbana para adecuar las vías a la gran cantidad de vehículos en uso. Como alternativa, podemos usar menos coches y más transportes alternativos que ocupan menos espacio en el tránsito, como por ejemplo la bicicleta.

En Brasil, las políticas públicas urbanas priorizan los desplazamientos por automóviles en sus inversiones, dejando de lado los transportes públicos y medios alternativos, causando así impactos negativos para la población y el medio ambiente.

La bicicleta es un vehículo individual, accesible a casi toda la población y no genera impactos negativos al medio ambiente como los vehículos automotores, además, es un medio de transporte flexible y económico, posee bajo consumo de energía, requiere poco espacio, reduce la emisión de contaminantes, contribuye a la reducción de los índices de ruido, contribuye al aumento del nivel de actividad física del usuario, favorece el aumento de la movilidad y calidad de vida e incluso contribuye a la inclusión social y, en casos de congestión, puede ser el medio de transporte más rápido.

La utilización de las bicicletas en las ciudades es un elemento fundamental para la implantación del concepto de Movilidad Urbana en la construcción de ciudades sostenibles, pues es un medio de transporte de fácil inserción en el contexto actual, además, reduce la degradación del medio ambiente y reduce costos con el transporte colectivo.

En Brasil existen cerca de 70 millones de bicicletas, es decir, uno de cada tres brasileños posee este medio de transporte, pero sólo 24 millones de personas las utilizan diariamente (el 12\% de la población) (ASSOCIAÇÃO NACIONAL E TRANSPORTES PÚBLICOS [ANTP], 2014). Esto ocurre debido 
a la resistencia de aceptación de la bicicleta como medio de transporte alternativo por parte de los usuarios siendo usada, en su mayoría, sólo para actividad de recreación o deporte profesional.

La inclusión de las bicicletas en los sistemas viales urbanos puede llevar al surgimiento de problemas en la ocupación de los espacios y dificultades estructurales, sin embargo esta inclusión es posible através de la distribución equitativa de las vías de acceso, educación para el tránsito e implantación de políticas públicas. Al final de las vías públicas ya están instituidas, siendo utilizadas por los automóviles, basta reducir las velocidades máximas permitidas, organizar el tráfico para fluir concomitantemente con las bicicletas y hacer campañas educativas para conductores y ciclistas a fin de que se tenga seguridad al traer en conjunto.

La ciudad de Uberlândia, MG, posee cerca de 676 mil habitantes y su infraestructura de transporte es organizada si se compara con los grandes centros urbanos de Brasil (INSTITUTO BRASILEIRO DE GEOGRAFIA E ESTATÍSTICA [IBGE], 2017). Aun así, la ciudad ya empieza a enfrentar el caos del tránsito, con grandes congestiones ubicados en el centro de la ciudad y en las áreas donde están las grandes empresas. El uso del transporte colectivo de esta ciudad es viable financieramente, pero se pierde mucho tiempo desplazándose en autobús, lo que hace la utilización del coche una alternativa más ágil, además del status de estabilidad financiera.

La población no ve la bicicleta como un medio de transporte económico y mucho menos como un factor importante para evitar la emisión de contaminantes. Por eso, se cree que esta falta de adhesión al medio de transporte alternativo es una cuestión cultural y / o falta de información sobre las aportaciones de la bicicleta a la salud de las personas, que en tiempos de tecnología, cada vez son más sedentarias. Por este motivo, el objetivo en este trabajo fue analizar la cultura de la utilización de la bicicleta como medio de transporte en el municipio de Uberlândia, MG, buscando demostrar la población los beneficios de su utilización para la salud del usuario y preservación del medio ambiente. 


\section{MATERIAL Y MÉTODOS}

Para el desarrollo de este estudio fue elegida la ciudad de Uberlândia, MG, ubicada en el Triángulo Minero y es el segundo mayor municipio del estado, con 676 mil habitantes (IBGE, 2017).

La ciudad posee infraestructura de transporte organizada, si se compara con los grandes centros urbanos de Brasil, habiendo recibido en los últimos años, la implantación de ciclovías y ciclofajas en determinadas áreas con la finalidad de incentivar el uso de los transportes alternativos y disminuir el flujo de vehículos automotores em el sistema viario.

El levantamiento de datos se efectuó a partir de observaciones de documentos e informaciones del Secretaría de Tráfico y Transportes (SETTRAN) relacionados con las ciclovías del municipio, así como del tránsito, del Ayuntamiento Municipal de Uberlândia y de la Universidad Federal de Uberlândia. La investigación siguió con la realización de un estudio exploratorio-descriptivo, con doble combinación de abordajes, de la investigación bibliográfica y cualitativa relacionada al uso de la bicicleta como medio de transporte alternativo.

Con el fin de complementar la recolección de datos y comprender las implicaciones culturales, se aplicó un cuestionario electrónico, disponible en plataforma on line, en el período de abril y mayo de 2017, con participación de 109 ciclistas. El cuestionario estaba compuesto de 14 cuestiones relacionadas con el perfil ciclístico de la población de Uberlândia (sexo, edad, escolaridad, franja de ingresos), además de cubrir cuestiones relacionadas al tiempo de uso de la bicicleta, ocurrencia de accidentes, dificultades encontradas para la utilización del medio de transporte alternativo, entre otras.

Los datos obtenidos fueron digitalizados y tabulados en hojas de trabajo del software de Microsoft Office Excel para formar la base de datos que fue analizada por medio de cálculo de medias simples y de porcentaje de forma cuantificable.

La recolección de datos (aplicación de cuestionario) fue una encuesta de opinión pública sin identificación, que dispensa la autorización del Comité de Ética de acuerdo con la resolución 510/2016 del CONEP. 


\section{RESULTADOS Y DISCUSIÓN}

\subsection{Análisis de los datos obtenidos por la aplicación del cuestionario en línea}

Con el fin de agregar datos a la investigación bibliográfica realizada relacionada al perfil del ciclista en Uberlândia, se puso a disposición un cuestionario online, durante los meses de abril y mayo de 2017.

Participaron de la investigación, 109 ciclistas, de los 52,3\% eran del sexo femenino y $47,7 \%$ del sexo masculino.

El grupo de edad de 35 a 44 años (24\%) fueron los que más respondieron al cuestionario, seguido por 24 a 34 años (23\%) y 45 a 54 años (22\%). Las investigaciones realizadas en Pelotas, RS y Brasilia (Región del Plan Piloto y Gama), corroboraron con ésta, en lo que se refiere a la edad media de los ciclistas (BACCHIERI; GIGANTE; ASSUNÇÃO, 2005; ARAUJO, 2016). Como nivel de escolaridad de estos ciclistas se destacó la Enseñanza media $(35,8 \%)$ y enseñanza superior $(30,3 \%)$, seguido por posgrado $(19,3 \%)$ y Enseñanza fundamental (14,7\%). Así como en un estudio realizado en São Carlos, SP, donde el grado de instrucción de la mayoría de los ciclistas era Enseñanza media (PROVIDELO; SANCHES, 2010).

La franja de ingreso predominante de los ciclistas de Uberlândia que participaron en la investigación, fue de 1 a 2 salarios mínimos, representando el 22\% del total (Figura 1). En Brasilia, en la región del Plan Piloto, según Araujo (2016), la utilización de bicicleta ocurre por la población de renta media (de 4 a 5 salarios mínimos), pues este local es una región donde se concentra la mayoría de la población de clase media del municipio. 
Uso de la bicicleta como transporte alternativo: implicaciones de la cuestión cultural en la

Figura 1 - Ingresos (en salario mínimo) de los ciclistas participantes de la investigación en Uberlândia, MG, de abril a mayo de 2017

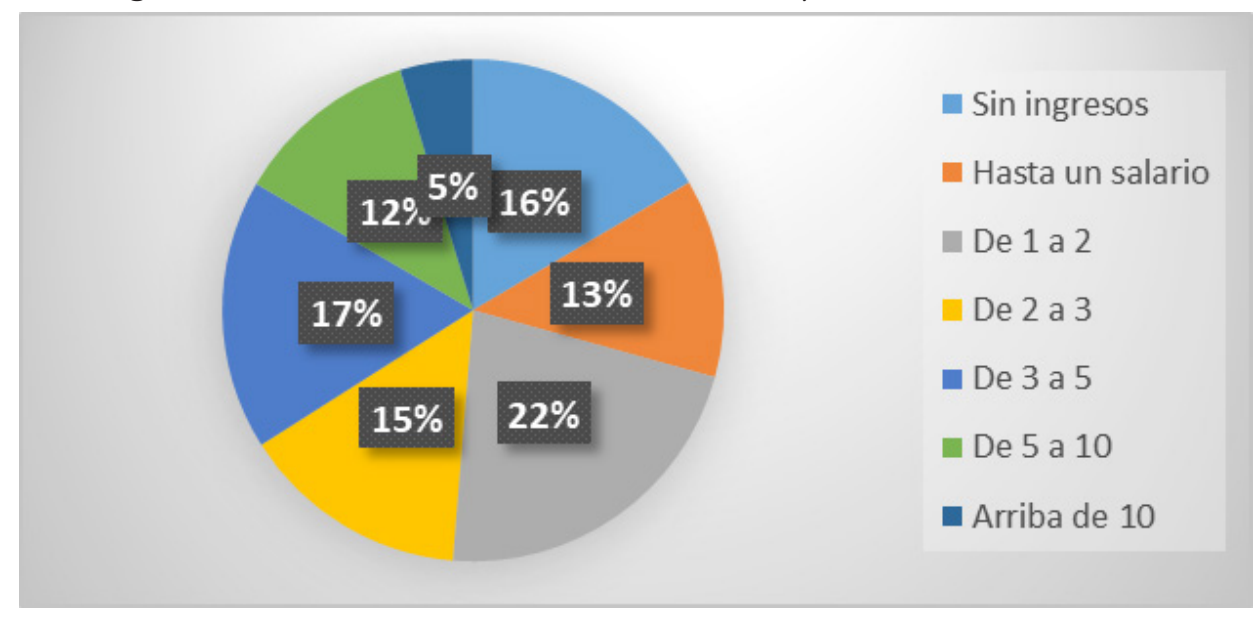

Funte: Autor propio.

La utilización de la bicicleta como medio de transporte alternativo sólo los fines de semana fue citada por el $61,5 \%$ de los encuestados. Hacen uso para trabajar, es decir, en días hábiles, el 22,9\%, y el 15,6\% relató hacer uso todos los días. La combinación entre bicicleta con otros medios de transporte fue citada por el 48,6\% de los entrevistados. En el caso de Brasil, la población de la región del Plan Piloto y de Gama suele utilizar la bicicleta todos los días, ya Providelo y Sanches (2010), detectaron uso sólo los fines de semana, corroborando los resultados como el de esta investigación.

Los ciclistas participantes en la investigación $(43,1 \%)$ dijeron hacer uso de la bicicleta a por lo menos 2 a 5 años, y el 33\% utilizan a poco tiempo (menos de 6 meses). Estos relataron también utilizarla por 11 a 30 minutos diarios (41,3\%), y el $22 \%$ por más de una hora. Con más coches en las calles, las condiciones del tránsito empeoran y los desplazamientos se vuelven más lentos no sólo para aquellos que utilizan sus propios vehículos, sino también para los que emplean transporte público. Los datos de la encuesta nacional por muestra de hogares (PNAD), del IBGE, evidencian que, en 2012, el tiempo promedio gastado en el desplazamiento casa / trabajo en las áreas metropolitanas de Brasil era de 40,8 minutos, un aumento de 4,4 minutos 
en con relación a 1992. Ya el número de personas que utilizan más de una hora de traslado al trabajo pasó del $14,6 \%$ al $18,6 \%$ en el mismo período (INSTITUTO DE PESQUISA ECONÔMICA APLICADA [IPEA], 2013). De este modo, la utilización de la bicicleta como medio alternativo sería válida, ya que posiblemente disminuiría el tiempo empleado en el desplazamiento para el trabajo.

La principal motivación para el uso de la bicicleta como transporte en Uberlândia, de acuerdo con el 62,4\% de los entrevistados, es la acción benéfica causada por ella debido a la realización de actividad física (Figura 2). Resultados similares fueron obtenidos por Bacchieri, Gigante y Assunção (2005), Providelo y Sanches (2010) y Araujo (2016). Un punto a destacar es la falta de concientización de los ciclistas de que el uso de la bicicleta no sólo está relacionado con la mejora de su calidad de vida, sino también la preservación del medio ambiente, ya que sólo el 9,2\% dijo que usan la bicicleta por ser un medio de transporte ambientalmente correcto. La población todavía no ha notado que usando la bicicleta diariamente dejan de usar vehículos automotores que emiten altos niveles de contaminantes.

Figura 2 - Motivación para utilizar la bicicleta como medio de transporte alternativo en Uberlândia, MG, 2017

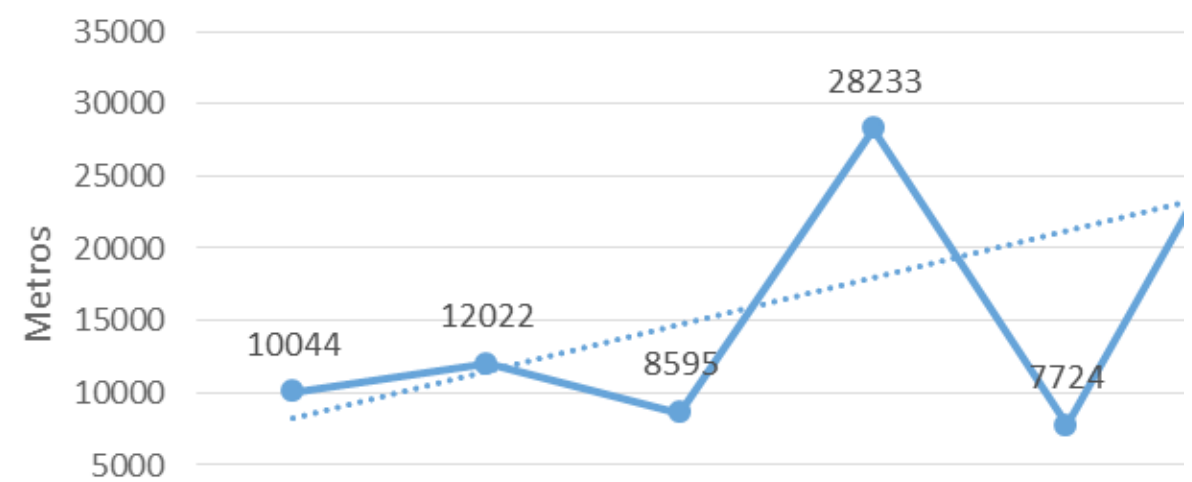

Funte: Autor propio. 
Según los ciclistas que respondieron al cuestionario, el principal problema enfrentado para el uso de la bicicleta como medio de transporte es la falta de infraestructura del Municipio (33\%), seguido por la falta de seguridad del tránsito $(30,3 \%)$ y la falta de respeto de los conductores de vehículos automotores para los ciclistas (23,9\%). Debido a esto, la mayoría $(40,4 \%)$ reivindica mejoras en la infraestructura cicloviaria del Municipio y también más medidas de seguridad en el tránsito (30,3\%). En Brasilia y Pelotas, la población está satisfecha con la malla cicloviaria e infraestructura local (BACCHIERI; GIGANTE; ASSUNÇÃO, 2005; PROVIDELO; SANCHES, 2010).

\subsection{Levantamiento de datos}

Los resultados obtenidos por medio del levantamiento de datos efectuado en el SETTRAN, en el Ayuntamiento Municipal de Uberlândia y en la Universidad Federal de Uberlândia, se describen a continuación.

La extensión de las ciclovías y ciclofaixas del Municipio de Uberlandia del período de 2000 a 2016 está representada en la Figura 3. Hasta el año 2000, Uberlândia poseía apenas 10.044 metros de ciclovías y ciclofaixas construidas. Debido al aumento de la demanda por la construcción de estas vías para un tránsito más seguro de los ciclistas, hubo un incremento sustancial en la construcción de las mismas, totalizando hasta 2016, 98.119 metros de ciclovías construidas en el Municipio, para una población de aproximadamente, 676 mil habitantes (IBGE, 2017). Según la ANTP (2014), en Brasil cerca del 12\% de la población utiliza la bicicleta como medio de transporte. Así, en Uberlandia se puede hacer una proyección de 81 mil ciclistas, para apenas 98 kilómetros de ciclovías y ciclofaixas construidas. 
Figura 3 - Extensión de las ciclovías, de Uberlandia, MG, en metros, del año 2000 a 2016

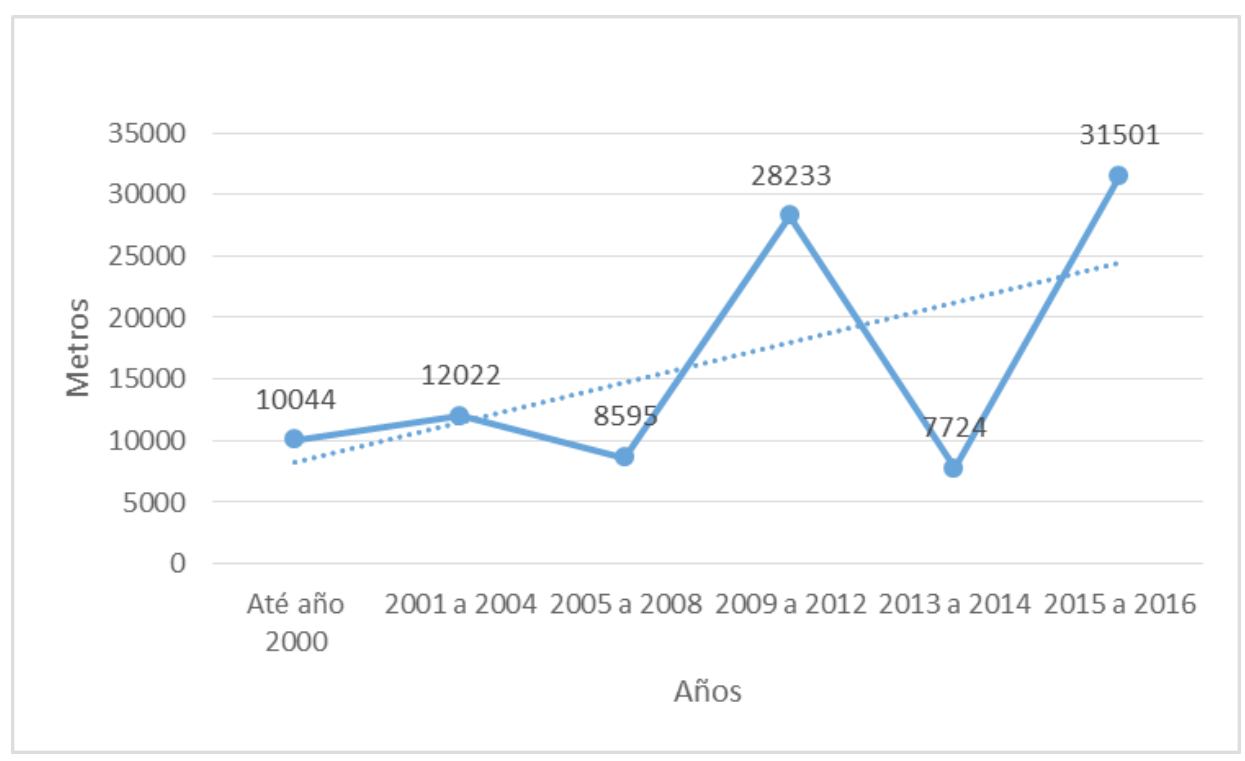

Funte: Autor propio.

Entre los años de 2013 y 2014, hubo una disminución acentuada en las construcciones debido a problemas internos del Ayuntamiento Municipal, pero las mismas fueron retomadas en 2015 y hasta 2016 oye un incremento del $400 \%$ en las construcciones cuando comparadas al período anterior. A pesar de presentar un crecimiento razonable, se percibe que su uso todavía es pequeño, teniendo como usuarios pocos trabajadores, deportistas, niños y algunos ciclistas eventuales.

La cuestión del desuso de las ciclovías y ciclofajas de Uberlandia, puede estar relacionado a la falta de divulgación de estas rutas alternativas por el Ayuntamiento Municipal, pues muchos ciudadanos desconocen la existencia de estas ciclovías y ciclofajas. En la ciudad, no hay placas señalando la ubicación de las mismas y el camino que ellas recorren hasta el destino final de cada una de ellas.

En la actualidad, según informaciones recolectadas en el Ayuntamiento Municipal, nuevos proyectos para la construcción de extensiones de las ciclovías y ciclofajas están siendo analizados, lo que incrementará el metraje 
ya construido, ya que poco a poco la bicicleta ha sido insertada en la vida de la población de Uberlândia, incluso que sea con el objetivo de ocio o practicar deportes.

Algunas regiones de la ciudad tienen proyecto en marcha, es decir, construcción de más ciclovías y ciclofajas, pero sin previsión de entrega de las obras. El sector oriental en la ciudad no tiene previsión para la construcción de ciclovías en los próximos años, lo que puede ser justificado por la falta de adhesión al uso de bicicletas como transporte alternativo o como forma de ocio en esta región. El sector Norte, Sur y Oeste de la ciudad tiene previsión para el aumento en la construcción de ciclovías y ciclofautas (Figura 4).

Figura 4 - Previsión de aumento de la extensión, en metros, por sectores, de las ciclovías de Uberlândia, MG, a partir de 2017

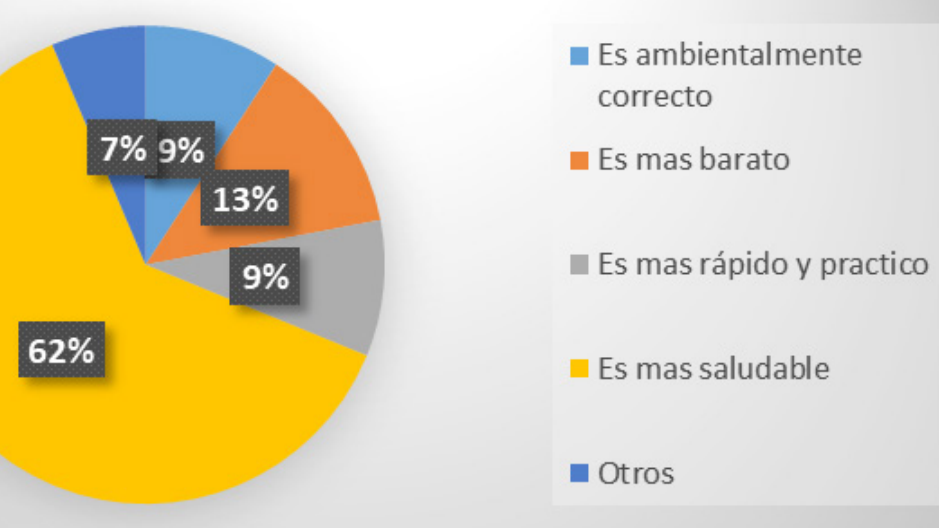

Funte: Autor propio.

La demora en la ampliación de las ciclovías y ciclofajas en Uberlândia está directamente relacionada al intercambio de gestión en la administración de la ciudad, además, el Municipio ha enfrentado dificultades financieras desde 2015, lo que inviabiliza la construcción de estas estructuras.

El mantenimiento y la construcción de ciclovías y ciclofijas es fundamental para la seguridad de los ciclistas. De los participantes, el 8,3\% ya relató la implicación en accidentes de tránsito con sus bicicletas en los últimos años. Además, hay registros de altos números de accidentes en hospitales 
públicos de la ciudad, en especial el Hospital de Clínicas de Uberlândia, que atiende casos de accidentes de tránsito. En las investigaciones cuyos ciclistas relataron óptimas condiciones de las ciclovías y ciclofosferas, tanto en relación a la conservación como la señalización, pocos se quejaron de ocurrencia de accidentes (BACCHIERI; GIGANTE; ASSUNÇÃO, 2005; PROIDELO; SANCHES, 2010).

Los accidentes, en su mayoría, son causados por la falta de atención de los ciclistas o de los conductores de vehículos automotores o incluso por falta de señalización en el tránsito, ya que la mayoría se produjo principalmente en el área donde se construyeron las ciclovías y ciclofajas. Los conductores todavía no están acostumbrados a las bicicletas que cruzan las calles, ya que no hay señalización adecuada para llamar la atención sobre los accidentes que puedan ocurrir. El tránsito compartido entre bicicletas y vehículos automotores es señalado como el principal factor de inseguridad, lo que facilita la ocurrencia de accidentes (SOUSA; BAHIA; CONSTANTINO, 2016).

De acuerdo con los datos proporcionados por el Pronto Socorro del Hospital de Clínicas de la Universidad Federal de Uberlândia, se ha elevado en la ciudad el número de accidentes involucrando a ciclistas en la ciudad, conforme a la Tabla 1. De 2011 a 2016, ocurrieron 845 accidentes involucrando a ciclistas, siendo en cruzamientos entre calles y ciclovías, debido a la mala señalización del local. Los estudios relacionados con accidentes de tránsito indican además la ocurrencia de subnotificaciones de accidentes con ciclistas, como estudio realizado en Pelotas, cuya tasa de subnotificación, comparando boletines de ocurrencia y atendimientos médicos, fue del 33,0\% (BASTOS; ANDRADE; SOARES, 2005), en Londrina, PR, Biazin y Rodrigues (2009) detectaron 8\% de subnotificaciones. 
Tabla 1 - Número de accidentes de tránsito involucrando a ciclistas en Uberlândia, MG, de 2011 a 2016 atendidos en el Hospital de las clínicas

\begin{tabular}{|c|c|c|c|c|c|c|c|c|}
\hline SEXO & EDAD & 2011 & 2012 & 2013 & 2014 & 2015 & 2016 & TOTAL \\
\hline \multirow[t]{6}{*}{ FEMENINO } & 0 a 20 años & 10 & 14 & 7 & 2 & 7 & 10 & 50 \\
\hline & 21 a 30 años & 4 & 5 & 5 & 4 & 7 & 4 & 29 \\
\hline & 31 a 40 años & 7 & 9 & 2 & 4 & 1 & 2 & 25 \\
\hline & 41 a 50 años & 9 & 0 & 2 & 4 & 1 & 5 & 21 \\
\hline & 51 a 60 años & 0 & 4 & 2 & 4 & 4 & 4 & 18 \\
\hline & Encima de 60 años & 0 & 1 & 0 & 0 & 1 & 0 & 2 \\
\hline TOTAL & & 30 & 33 & 18 & 18 & 21 & 25 & 145 \\
\hline \multirow[t]{6}{*}{ MASCULINO } & 0 a 20 años & 54 & 74 & 43 & 26 & 24 & 40 & 261 \\
\hline & 21 a 30 años & 41 & 38 & 20 & 11 & 8 & 19 & 137 \\
\hline & 31 a 40 años & 17 & 26 & 20 & 12 & 13 & 13 & 101 \\
\hline & 41 a 50 años & 14 & 22 & 15 & 14 & 5 & 13 & 83 \\
\hline & 51 a 60 años & 2 & 22 & 14 & 9 & 9 & 7 & 63 \\
\hline & Encima de 60 años & 8 & 10 & 13 & 6 & 9 & 9 & 55 \\
\hline TOTAL & & 136 & 192 & 125 & 78 & 68 & 101 & 700 \\
\hline $\begin{array}{c}\text { TOTAL } \\
\text { PERÍODO }\end{array}$ & & 166 & 225 & 143 & 96 & 89 & 126 & 845 \\
\hline
\end{tabular}

Fuente: Pronto Socorro - Hospital das Clínicas [adaptado] (2017).

El grupo de edad de mayor atención de accidentes de tránsito ciclísticos de 2011 a 2016 fue de hasta 20 años de edad. En el levantamiento de datos del cuestionario, se observó que la escolaridad de los participantes fue, en su mayoría, estudiantes universitarios o de la escuela secundária, encuadrándose en el grupo de edad citada. Posiblemente este público utiliza el medio de transporte para desplazarse a la Universidad o a la escuela. En una encuesta realizada por Souza, Bahia y Constantino (2016), analizando el banco de datos de la VIVA encuesta (Sistema de Vigilancia de Violencias y Accidentes del Ministerio de Salud) realizado en 2014, relataron que el grupo de edad de mayor ocurrencia de accidentes son personas de 20 a 39 años, así como en el que el 50\% de los accidentados tenían entre 17 y 31 años (ANDRADE; MELO, 2000). 
Se verificó también que, de acuerdo con el aumento del grupo de edad, el número de accidentes fue disminuyendo, tanto para el sexo masculino y para el femenino. Los ciclistas masculinos también presentaron un porcentaje alto en accidentes en comparación con los ciclistas femeninos. Las investigaciones realizadas através de entrevistas a ciclistas, demostró la predominancia de ciclistas del sexo masculino, de esta forma, se cree que éste es el factor que influye en un número mayor de accidentes con hombres (BACCHIERI; GIGANTE; ASSUNÇÃO, 2005; PROIDELO; SANCHES, 2010).

El predominio de accidentes que involucran a hombres también se ha encontrado en otros estudios, como el de Gawryszewsk et al. (2005), en São Paulo, el cual apuntó que los hombres concentraron el 74,2\% del total de atendimientos, así como la encuesta realizada por Souza, Bahia y Constantino (2016), que relataron que la mayoría de los accidentes ocurren con hombres, denotando que los mismos tienen un 59,0\% más probabilidades de ser víctimas de accidentes al traer en bicicleta, en comparación con las mujeres.

A pesar de la ocurrencia de accidentes, muchos ciclistas hacen uso de las ciclovías y ciclofajas. Así, la inversión en nuevos proyectos para la construcción, revitalización, ampliación y señalización de ciclovías y ciclofajas debe ser realizada, y también se verifica la necesidad de mejoras en los proyectos de construcción y señalización de las mismas, lo que puede disminuir el número de accidentes que involucra a ciclistas en Uberlândia. La necesidad de mejora ya fue verificada por otros autores, como por el estudio realizado en Maringá, $\mathrm{PR}$, que evidenció mala señalización, con placas de indicación de la ciclovía en algunos puntos apenas, calzado precario, sin rampas de acceso, falta de placas de señalización alertando para el cruce de vehículo y problema de iluminación pública, lo que afecta directamente la seguridad del local (SIMONI et al., 2015).

Pezzuto (2002) ha identificado algunos factores que contribuyen a la decisión de usar la bicicleta como modo de transporte, son ellos: seguridad, distancia del viaje, conveniencia, tiempo de viaje, hábito, costo, motivación, clima, infraestructura y topografía. Además, muchas ciudades brasileñas optaron por invertir en la construcción de ciclovías y ciclofautas para aumentar la utilización de este medio transporte alternativo con el 
Uso de la bicicleta como transporte alternativo: implicaciones de la cuestión cultural en la preservación del medio ambiente

objetivo de disminuir la contaminación provocada por el gran número de coches circulando en las calles.

La concientización de la población en cuanto a los daños al medio ambiente y a los beneficios para la salud del uso de la bicicleta como medio de transporte aún no están bien claros y deben ser trabajados, ya que la utilización de la bicicleta se considera un vehículo sostenible por no poner en peligro la calidad de vida y los ecosistemas, además de traer mejoría de movilidad urbana a los usuarios.

Las necesidades de cambios en relación a los planes de tráfico de la ciudad, principalmente en relación al uso de bicicletas como transporte alternativo, también deberán ser analizadas y efectuadas. Para ello, es necesario que las políticas públicas del Municipio inviertan en divulgación y persuasión en relación al uso de las ciclovías y ciclofajas, lo que puede auxiliar en el cambio de hábitos culturales de la población en relación al uso diario de la bicicleta, no sólo como objeto de deporte pero como medio de transporte alternativo.

La implantación de bicicletarios en ciclovías ya existentes, terminales de transportes urbanos, centros comerciales, Universidades, escuelas y otros lugares, e incluso la adquisición de nuevos modelos de autobuses urbanos característicos a transportar bicicletas, también debe ser analizada para estimular el uso cotidiano de la bicicleta por la población.

Además de la infraestructura para la circulación, los bicicletarios deben ser diseñados para el confort y seguridad de aquellos que están contribuyendo a la mejora del medio ambiente, con el objetivo de atender a las características específicas de las regiones, como protección contra robo, vandalismo e intemperie. También debe existir en estos lugares la posibilidad de movimiento seguro de los ciclistas a su entrada y salida, principalmente cuando están instalados cerca de los puntos de conexión con ómnibus.

El uso del coche a menudo se convierte en una necesidad, pero la bicicleta puede convertirse en un aliada importante en relación con la salud, la movilidad y la preservación del medio ambiente, por lo que es necesario establecer métodos educativos relacionados con la concienciación cultural de la población. La promoción del uso de la bicicleta pasó a ser una de las 
metas de la Organización Mundial de la Salud tanto por la necesidad de reducción de contaminantes en el ambiente de las ciudades debido a la elevada motorización, como por la promoción de la salud, que su utilización como medio de transporte puede representar la a medio y largo plazo (DORA; PHILLIPS, 2000).

\section{CONCLUSIONES}

Por medio del estudio realizado, fue posible verificar la deficiencia de infraestructura cicloviária en Uberlândia, lo que puede haber sido uno de los factores que ocasionaron un número elevado de accidentes involucrando a ciclistas ya que la mayoría ocurrió en cruzamientos entre calle y ciclovía. Incluso con las vulnerabilidades encontradas en el sector, la bicicleta todavía se considera uno de los medios de transporte más sanos, siempre y cuando se garantizan los aspectos de seguridad. De esta forma, quedó evidente la necesidad de discusiones sobre acciones que apunten a aumentar el uso de la misma como medio de transporte alternativo por la población, para mejorar la movilidad urbana, salud pública y medio ambiente.

También fue notoria la necesidad de implantar medidas para disminuir las ocurrencias de accidentes de tránsito involucrando a ciclistas, tales como reestructuración de programas de políticas públicas, mejora y aumento de las ciclovías y ciclofajas, mejora de la fiscalización de las leyes de tránsito, además de la obligatoriedad de la utilización de los equipos de seguridad son factores fundamentales para lograr esta meta.

\section{REFERENCIAS}

ANDRADE, S. M.; MELLO, J. M. H. P. Características das vítimas por acidentes de transporte terrestre em município da Região Sul do Brasil. Revista de Saúde Pública, São Paulo, v. 34, n. 2, p. 149-56, abr. 2000.

ARAUJO, M. C. V. Estudo de caso: levantamento do perfil dos usuários das ciclovias de três Regiões Administrativas do Distrito Federal. 2016. Documento de Conclusión del Curso (Graduación en Ingeniería Civil)- Centro Universitário de Brasília (UniCEUB), Brasília, 2016. 
Uso de la bicicleta como transporte alternativo: implicaciones de la cuestión cultural en la preservación del medio ambiente

ASSOCIAÇÃO NACIONAL E TRANSPORTES PÚBLICOS (ANTP). Sistema de Informações da Mobilidade Urbana. Relatório Geral 2012. Jul. 2014. Disponible en: http:// files-server.antp.org.br/_5dotSystem/download/dcmDocument/2014/08/01/ CB06D67E-03DD-400E-8B86-D64D78AFC553.pdf. Acceso el: 12 oct. 2019.

BACCHIERI, G.; GIGANTE, D. P.; ASSUNÇÃO, M. C. Determinantes e padrões de utilização da bicicleta e acidentes de trânsito sofridos por ciclistas trabalhadores da cidade de Pelotas, Rio Grande do Sul, Brasil. Cadernos de Saúde Pública, Rio de Janeiro, v. 21, n. 5, p. 1499-508, sept./oct. 2005.

BASTOS, Y. G. L.; ANDRADE, S. M.; SOARES, D. A. Características dos acidentes de trânsito e das vítimas atendidas em serviço pré-hospitalar em cidade do Sul do Brasil, 1997/2000. Cadernos de Saúde Pública, Rio de Janeiro, v. 21, n. 3, p. 81522, 2005.

BIAZIN, D. T.; RODRIGUES, R. A. Perfil dos idosos que sofreram trauma em LondrinaParaná. Revista da Escola de Enfermagem da USP, São Paulo, v. 43, n. 3, p. 602-8, 2009.

DORA, C.; PHILLIPS, M. Transport, environment and health. Copenhagen: WHO Regional Publications, European Series, 2000. Disponible en: http://www.euro. who.int/_data/assets/pdf_file/0003/87573/E72015.pdf. Acceso el: 20 nov. 2017.

GAWRYSZEWSKI, V. P. et al. Land transport injuries among emergency department visits in the state of São Paulo, in 2005. Revista de Saúde Pública, São Paulo, v. 43, n. 2, p. 275-82, 2009.

INSTITUTO BRASILEIRO DE GEOGRAFIA E ESTATÍSTICA (IBGE). Diretoria de Pesquisas, Coordenação de População e Indicadores Sociais. Estimativas da população residente com data de referência 10 de julho de 2017. Disponible en: www.cidades. ibge.gov.br/xtras/perfil.php?codmun=317020. Acceso el: 4 nov. 2019.

INSTITUTO DE PESQUISA ECONÔMICA APLICADA (IPEA). Indicadores de mobilidade urbana da Pnad 2012. Brasília, 2013. (Comunicado do IPEA; n. 161). Disponible en: http://www.ipea.gov.br/portal/images/stories/PDFs/comunicado/131024_ comunicadoipea161.pdf. Acceso el: 5 nov. 2019.

PEZZUTO, C. C. Fatores que influenciam o uso de bicicleta. 2002. 146 f. Disertación (Mestrado em Engenharia Urbana)- Universidade Federal de São Carlos (UFSCAR), São Carlos, SP, 2002. 
PROVIDELO, J. K.; SANCHES, S. P. Percepções de indivíduos acerca do uso da bicicleta como modo de transporte. Revista Transportes, São Paulo, v. XVIII, n. 2, p. 53-61, jun. 2010.

SIMONI, J. H.; FIORELLI, M. N.; ALENCAR, J. L. S.; ANGELIS NETO, G. Conflito entre pedestre e ciclovia: um estudo de caso da Avenida Mandacarú em Maringá - PR. Revista Eletrônica em Gestão, Educação e Tecnologia Ambiental, Santa Maria, RS, v. 19, n. 2, p. 587-94, maio/ago. 2015.

SOUSA, C. A. M.; BAHIA, C. A.; CONSTANTINO, P. Análise dos fatores associados aos acidentes de trânsito envolvendo ciclistas atendidos nas capitais brasileiras. Ciência \& Saúde Coletiva, Rio de Janeiro, v. 21, n. 12, p. 3683-90, 2016. 